\title{
Case Reports in Neurology
}

\section{Recurrent Stroke in a Young Patient with Embolic Stroke of Undetermined Source and Patent Foramen Ovale: Quo Vadis?}

\author{
Katharina Feil ${ }^{a, b}$ Johanna Heinrich ${ }^{a}$ Aenne S. von Falkenhausen ${ }^{c, d}$ \\ Regina Becker ${ }^{a, b} \quad$ Clemens Küpper ${ }^{a} \quad$ Katharina Müller ${ }^{a}$ \\ Dennis C. Thunstedt ${ }^{a}$ Moritz F. Sinner ${ }^{c, d}$ Stefan Kääb ${ }^{c, d} \quad$ Lars Kellert $^{a}$ \\ aDepartment of Neurology, Ludwig Maximilian University, Munich, Germany; \\ ${ }^{b}$ German Center for Vertigo and Balance Disorders, Ludwig Maximilian University, \\ Munich, Germany; 'Department of Medicine 1, Ludwig Maximilian University, \\ Munich, Germany; ${ }^{\mathrm{d}}$ German Centre for Cardiovascular Research (DZHK), partner site: \\ Munich Heart Alliance, Munich, Germany
}

\section{Keywords}

Embolic stroke of undetermined source - Patent foramen ovale closure - Atrial fibrillation .

Insertable cardiac monitor

\section{Abstract}

So far, there has been no generally accepted diagnostic and therapeutic algorithm for patients with embolic stroke of undetermined source (ESUS). As recent clinical trials on secondary stroke prevention in ESUS did not support the use of oral anticoagulation and the concept of ESUS comprises heterogeneous subgroups of patients, including a wide age range, concomitant patent foramen ovale (PFO), variable cardiovascular risk factors as well as a variable probability for atrial fibrillation (AF), an individualized clinical approach is needed. In this context we here present a case of recurrent stroke in a young patient with ESUS and PFO. During treatment according to our Catch-up-ESUS registry study, prolonged cardiac monitoring diagnosed $A F$, and PFO closure was omitted.

(C) 2020 The Author(s)

Published by S. Karger AG, Basel 


\section{Introduction}

Recently, the topic of optimal secondary prophylaxis in embolic stroke of undetermined source (ESUS) patients has been discussed, especially in the context of NAVIGATE ESUS [1] and RE-SPECT ESUS [2] comparing the efficacy and safety of oral anticoagulation (OAC) with rivaroxaban [1] or dabigatran [2] to treatment with acetylsalicylic acid (ASA). There were no differences in stroke recurrence rates per year (4.7\% rivaroxaban vs. $4.7 \%$ ASA and $4.1 \%$ dabigatran vs. $4.8 \%$ ASA) $[1,2]$. However, patients treated with rivaroxaban had significantly higher annual bleeding rates (1.8 vs. $0.7 \%$ ) [1]. While a consensus on secondary stroke prevention in ESUS patients has yet to be reached, OAC is not recommended and all ESUS patients receive ASA, even if the risk of atrial fibrillation (AF) may be increased. Moreover, recommendations about searching for $\mathrm{AF}$ are lacking, although it was shown that an insertable cardiac monitor (ICM, event recorder) detects $\mathrm{AF}$ in about $40 \%$ of a high-risk population within one year after ESUS [3]. Another unresolved question is the role of a relevant persistent patent foramen ovale (PFO) in ESUS patients. A common finding in about $25 \%$ of the general population, PFO prevalence is higher in up to $50 \%$ of ESUS patients depending on patient age [4]. According to the recently published guidelines of the German Society of Neurology, all "patients between 16 and 60 years of age with a cryptogenic ischemic stroke and PFO with moderate or pronounced shunt are recommended a PFO closure" [5]. These national recommendations are also mirrored internationally [6]. On the other hand, all of these guidelines do not cover the extent of neurological and cardiological assessment required prior to PFO closures, especially regarding the diagnostic procedures to rule out $\mathrm{AF}$, i.e., by prolonged electrocardiogram (ECG) screening. Therefore, there is no comment on the need for screening for AF.

\section{Case Report}

A 51-year-old female with no relevant past medical history was admitted to our neurological department in 2018 with acute onset of double vision. Magnetic resonance imaging (MRI) showed ischemia in the area of the right median thalamus (Fig. 1a). Diagnostic workup was performed according to our in-house treatment algorithm according to the Catch-upESUS registry (for further information on Catch-up-ESUS, see [7]). Catch-up-ESUS is an openlabel, academic, prospective, monocentric, observational registry study that started in January 2018. Clinical data of all consecutive ESUS patients $\geq 18$ years old treated in our hospital who have given written informed consent are collected and observed [7]. The treatment algorithm in Catch-up-ESUS includes ultrasound examination of the brain vessels, cardiac examination with ECG monitoring for at least 72 hours, transthoracic and transesophageal echocardiography, blood tests, and assessment of AF-related risk markers [7].

Transcranial contrast-enhanced ultrasound ("bubble test") showed left-right shunt and transesophageal echocardiography confirmed a relevant PFO with 33 bubbles (Fig. 1d). A Risk of Paradoxical Embolism (RoPE) score of 6 at this time point suggested an intermediate causality between the PFO and ESUS [8]. Thus, according to the treatment algorithm within Catchup-ESUS, the patient was treated with ASA as secondary stroke prevention. In addition, an ICM was implanted. PFO closure was recommended if AF was ruled out during the following 3-6 months. In January 2019, the patient was readmitted with acute onset of expressive aphasia and hemiparesis on the right side (NIHSS 3, modified Rankin scale 2). Multimodal computed tomography (CT) imaging with CT angiography showed middle cerebral artery occlusion on the left side with mismatch in CT perfusion (Fig. 1c). Our patient was treated with intravenous 
thrombolysis within 70 min and improved clinically (modified Rankin scale 0 at discharge). Deep vein thrombosis had been ruled out both times (D-dimers 0.8 and $0.7 \mathrm{mg} / \mathrm{dL}$, normal range $<0.5 \mathrm{mg} / \mathrm{dL}$ ); venous ultrasound as standard imaging to rule out deep vein thrombosis was performed as part of the Catch-up-ESUS treatment algorithm and was unremarkable. A few days after recurrent stroke, AF was detected over a time period of $30 \mathrm{~s}$. PFO closure was omitted. Instead, the patient was treated with lifelong OAC.

\section{Discussion/Conclusion}

Regarding our case, the treatment in accordance with the guidelines would have been an immediate PFO closure without extensive cardiac rhythm monitoring and subsequent AF detection. As AF is considered to be the etiology of recurrent stroke, our case demonstrates the need for intensive monitoring for AF in ESUS patients and concomitant PFO. This can prevent unnecessary PFO closure procedures in patients in whom AF is detected afterwards with an indication for lifelong OAC. In addition, $\mathrm{PFO}$ closure can cause $\mathrm{AF}$ as a periprocedural complication in about $5 \%$ of patients, and development of AF is probably much more likely over the following years and decades, but data regarding this point are sparse [9]. Therefore, ICM implantation before PFO closure should be considered. The diagnostic challenge and importance of new guideline recommendations is reflected by this case and the Catch-up-ESUS registry study [7]. In our Catch-up-ESUS registry study [7], a standardized search for AF is implemented for up to 6 months using ICM prior to an interventional PFO closure. Especially as PFO closure is invasive, possibly resulting in complications, an undesired closure may be avoided in patients with an indication for lifelong OAC. Additionally, the group of ESUS patients $<60$ years of age presented in the discussed clinical trials is very heterogeneous, ranging from young patients with PFO and without any cardiovascular risk factors as discussed in our case report to middle-aged and old patients with a high cardiovascular risk profile. In summary, in stroke and PFO, prolonged cardiac monitoring for the detection of AF should be considered before PFO closure.

\section{Acknowledgement}

We thank Katie Göttlinger for copy editing the manuscript.

\section{Statement of Ethics}

The registry study Catch-up-ESUS has been approved by the ethics committee at the Ludwig Maximilian University (LMU), Munich, Germany (project number 17-685) and has been registered at www.clinicaltrialsregister.gov (NCT03820375, registered on January 14, 2019). The registry study is conducted in accordance with the Declaration of Helsinki. The patient has given informed consent to take part in the registry study and to the publication of individual treatment details (anonymized). 


\section{Disclosure Statement}

A.S. von Falkenhausen was supported by the Clinician Scientist Program in Vascular Medicine (PRIME) funded by the German Research Foundation (DFG) and the Medical Faculty of the LMU, Munich, Germany. The other authors have no conflicts of interest to declare.

\section{Funding Sources}

There were no funding sources for the registry study and the case report.

\section{Author Contributions}

All authors have read and approved the case report before submission.

K.F.: drafting/revising of the manuscript for content, including medical writing, registry study concept, design, interpretation of clinical data and acquisition of data, development of clinical algorithm.

J.H., C.K., R.B., K.M., D.C.T.: revising manuscript for content, including medical writing.

A.S.F.: revising manuscript for content, including medical writing, acquisition of data.

S.K.: revising manuscript for content, including medical writing, acquisition of data, study concept, development of clinical algorithm.

M.F.S.: revising manuscript for content, including medical writing, acquisition of data, study concept, development of clinical algorithm.

L.K.: drafting/revising of the manuscript for content, including medical writing, study concept, design, interpretation of data, acquisition of data, development of clinical algorithm.

\section{References}

1 Hart RG, Sharma M, Mundl H, Kasner SE, Bangdiwala SI, Berkowitz SD, et al.; NAVIGATE ESUS Investigators. Rivaroxaban for Stroke Prevention after Embolic Stroke of Undetermined Source. N Engl J Med. 2018 Jun;378(23):2191-201.

2 Diener HC, Sacco RL, Easton JD, Granger CB, Bernstein RA, Uchiyama S, et al.; RE-SPECT ESUS Steering Committee and Investigators. Dabigatran for Prevention of Stroke after Embolic Stroke of Undetermined Source. N Engl J Med. 2019 May;380(20):1906-17.

3 Poli S, Diedler J, Härtig F, Götz N, Bauer A, Sachse T, et al. Insertable cardiac monitors after cryptogenic stroke-a risk factor based approach to enhance the detection rate for paroxysmal atrial fibrillation. Eur J Neurol. 2016 Feb;23(2):375-81.

4 Kent DM, Dahabreh IJ, Ruthazer R, Furlan AJ, Reisman M, Carroll JD, et al. Device Closure of Patent Foramen Ovale After Stroke: Pooled Analysis of Completed Randomized Trials. J Am Coll Cardiol. 2016 Mar;67(8):907-17.

5 Diener HC, Gerloff C, Thaler DE, Wöhrle J. Closure of Patent Foramen Ovale and Cryptogenic Stroke: unresolved Issues. Curr Neurol Neurosci Rep. 2018 Oct;18(12):92.

6 Kuijpers T, Spencer FA, Siemieniuk RA, Vandvik PO, Otto CM, Lytvyn L, et al. Patent foramen ovale closure, antiplatelet therapy or anticoagulation therapy alone for management of cryptogenic stroke? A clinical practice guideline. BMJ. 2018 Jul;362:k2515.

7 Feil K, Heinrich J, Küpper C, Müller K, Laub C, von Falkenhausen AS, et al. Catch-up-ESUS: Follow-up in embolic stroke of undetermined source (ESUS) in a prospective, open-label, observational study: study protocol and initial baseline data. BMJ Open. 2019;9(12):e031716.

8 Kent DM, Ruthazer R, Weimar C, Mas JL, Serena J, Homma S, et al. An index to identify stroke-related vs incidental patent foramen ovale in cryptogenic stroke. Neurology. 2013 Aug;81(7):619-25. 


\section{Case Reports in Neurology}

\begin{tabular}{l|l}
\hline Case Rep Neurol 2020;12:45-49 \\
\hline DOI: 10.1159/000505180 & $\begin{array}{l}\text { @ 2020 The Author(s). Published by S. Karger AG, Basel } \\
\text { www.karger.com/crn }\end{array}$ \\
\hline
\end{tabular}

Feil et al.: Recurrent Stroke in a Young Patient with Embolic Stroke of Undetermined Source and Patent Foramen Ovale: Quo Vadis?

9 Søndergaard L, Kasner SE, Rhodes JF, Andersen G, Iversen HK, Nielsen-Kudsk JE, et al.; Gore REDUCE Clinical Study Investigators. Patent Foramen Ovale Closure or Antiplatelet Therapy for Cryptogenic Stroke. N Engl J Med. 2017 Sep;377(11):1033-42.
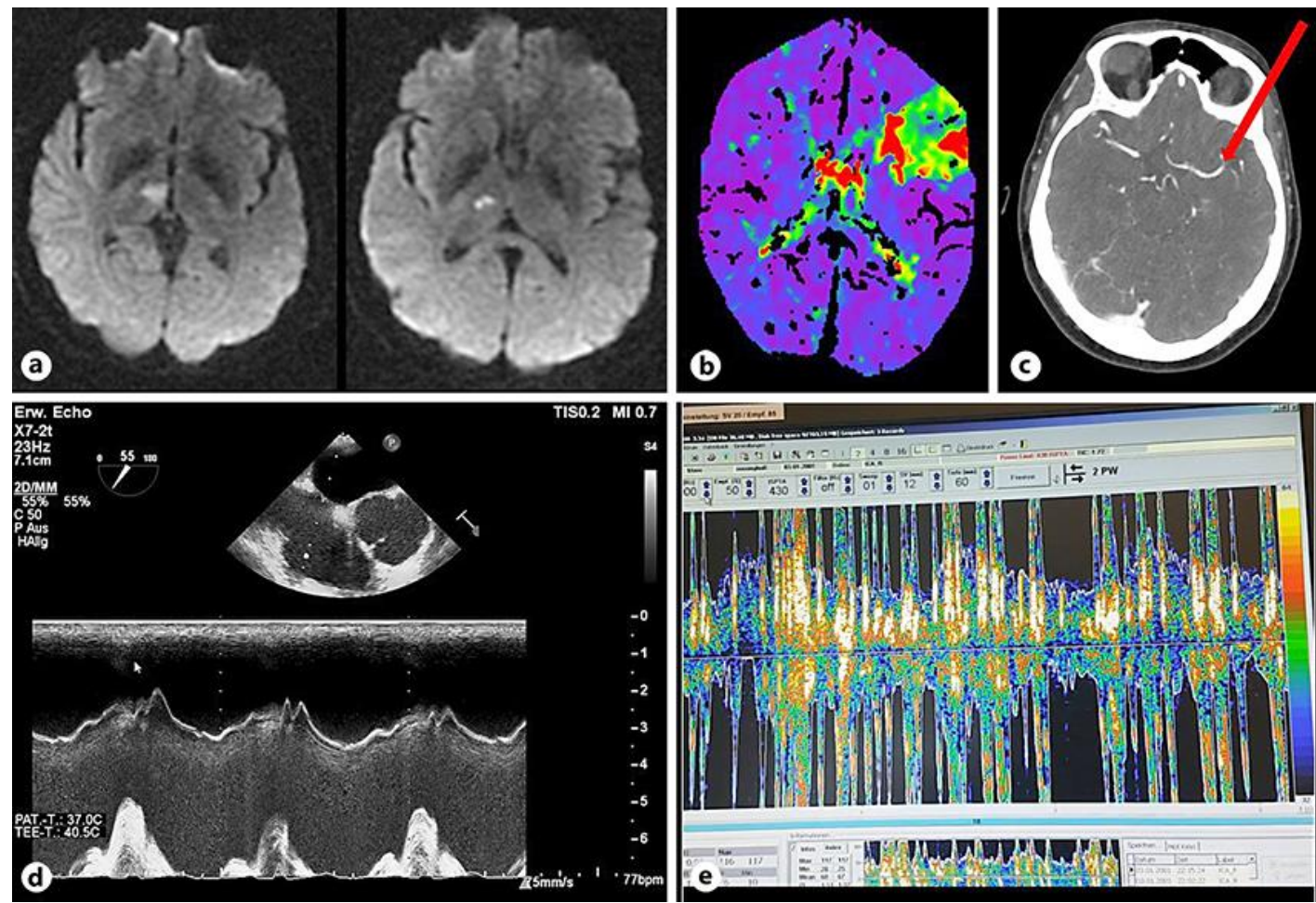

Fig. 1. a Cerebral MRI with ischemia in the right median thalamus at the first admission to hospital. b Computed tomography (CT) imaging with CT perfusion (time to drain) with decreased blood flow in the media flow area on the left side at the second admission to hospital. c CT imaging with CT angiography showing occlusion of the middle cerebral artery on the left side. $\mathbf{d}$ Transesophageal echocardiography with moderate shunt. e Transcranial contrast-enhanced ultrasound ("bubble test") showing left-right shunt. 